\title{
Percutaneous Cholecystostomy as an Alternative to Cholecystectomy in High-Risk Patients with Acute Cholecystitis.
}

\author{
Salih Tosun', Oktay Yener ${ }^{1}$, Ozgur Ekinci' ${ }^{1}$ Aman Gapbarov ${ }^{1}$, Murat \\ Asık $^{2}$, Tunc Eren' ${ }^{1}$, Orhan Alimoglu1 \\ https://doi.org/10.32391/ajtes.v3i2.39
}

\begin{abstract}
Background: Cholecystectomy is the standard treatment for patients with acute cholecystitis. On the other hand, percutaneous cholecystostomy (PC) is an alternative for patients at high-risk for surgery. The aim of this study was to evaluate the clinical outcomes of PC.

Methods: Surgically high-risk patients with acute cholecystits having undergone PC at our institution between January 2014 - January 2017 were evaluated. Data including the indications for PC, route of insertion, technical success, clinical improvement, length of hospital stay, mortality rates, procedure related complications, subsequent admissions and performance of interval cholecystectomy were recorded and analyzed.

Results: The study group consisted of 30 patients with a mean age of 75.3 ( range: 4999) years. The indications for PC were acute calculous cholecystitis in $28(93.3 \%)$ and acalculous cholecystitis in $2(6.7 \%)$ patients. All procedures were performed via the transhepatic route. Twenty-five PCs $(84.4 \%)$ resulted in clinical improvement within five days. A repeated PC was performed in two (6.7\%) patients. Seven (23.3\%) patients underwent a subsequent cholecystectomy after 6 weeks. An emergent cholecystectomy was performed in one $(3.3 \%)$ patient. Five $(16.7 \%)$ patients died from underlying comorbid diseases, unrelated to the biliary system, during the follow-up.

Conclusions: Patients with acute cholecystitis were promptly relieved from their symptoms following PC. There were only minor complications following the procedure and an interval cholecystectomy was necessary in only $23.3 \%$ of the patients. PC is a safe alternative to choecystectomy in high-risk patients with acute cholecystitis with satisfactory results.

Keywords: Cholecystostomy, cholecystitis, general surgery
\end{abstract}

*Corresponding author: Orhan Alimoglu

$=凶$ E-mail: orhanalimoglu@gmail.com

${ }^{1}$ Department of General Surgery, Faculty of Medicine, Istanbul Medeniyet University, Istanbul, Turkey

2 Department of Radiology, Faculty of Medicine, Istanbul Medeniyet University, Istanbul, Turkey 


\section{Full Text}

\section{Introduction}

Acute cholecystitis is a commonly encountered disease in surgical departments and the number of treated patients is increasing as the population ages. The first cholecystotomy was performed by Bobbs in 1867 and the first cholecystectomy by Langenbuch in 1874 1. Standard treatment for acute cholecystitis has for many years been laparoscopic cholecystectomy (LC) in the acute phase. Cholecystectomy remains the reference standard for treating acute cholecystitis, but the perioperative mortality rates in the elderly or critically ill population are reported to be as high as $19 \%$. Therefore, cholecystostomy is regarded as a safe alternative with a good therapeutic response, especially in surgically high-risk populations ${ }^{2}$.

The scientific evidence for advocating any specific therapy for acute calculous cholecystitis is sparse. Even the Tokyo guidelines for acute cholecystitis are mainly based on opinions among wellknown surgeons and not on highquality scientific evidence ${ }^{3}$. Some researchers have also questioned the necessity for surgery in acute cholecystitis, even in the long term. Conservative treatment with antibiotics and percutaneous drainage are reported as an adequate alternative to surgery, although most authors recommend delayed cholecystectomy in these cases 4 .

The first ultrasound guided percutaneous cholecystostomy (PC) was performed in a jaundiced patient in 1979 by Elyaderani and Gabriele. In
1982 Radder, followed a year later by Elyaderani et al, attempted the procedure in patients with acute cholecystitis ${ }^{5}$. The method was further developed by Shaver et al, and became established as a minimally invasive alternative in patients not considered fit for cholecystectomy. PC can be used as a treatment for acute cholecystitis in the elderly or critically ill patients, allowing subsequent elective cholecystectomy with minimal mortality. Additionally, in some cases, PC may be the definitive treatment for gallstone disease ${ }^{6}$.

The purpose of this study was to evaluate the clinical outcomes and possible benefits of PC in high-risk patients with acute cholecystitis.

\section{Methods}

The medical records of surgically highrisk patients with either calculous, or acalculous acute cholecystitis having undergone PC (the PC group) between January 2014 and January 2017 at our institution were reviewed retrospectively. Data including age, sex, indications for cholecystostomy, route of insertion, technical success, clinical improvement, mortality rate, complications, subsequent admissions and performance of interval cholecystectomy were evaluated.

PC was performed under fluoroscopic and ultrasonographic guidance in the Department of Interventional Radiology. The transhepatic route was preferred in order to reduce the risk of bile leakage and to facilitate rapid tract maturation. Lidocaine was injected as 
a local anesthetic to the application area. The Seldinger tecnique was used to access the gallbladder. An 18-gauge needle was introduced through the gallbladder lumen under ultrasonographic guidance. Several millilitres of diluted contrast agent was injected to opacify the gallbladder lumen under fluoroscopy in order to confirm intraluminal position and patency of the cystic duct. A 0.035 inch $75 \mathrm{~cm}$ Amplatz super-stiff guidewire was inserted through the needle. Dilatation of the tract was performed with $6 \mathrm{~F}$ and $8 \mathrm{~F}$ dilators respectively. An $8 \mathrm{~F}$ pigtail catheter (Flexima ${ }^{\circledR}$, Boston Scientific, USA) was inserted into the gallbladder. If purulent bile was aspirated, a $10 \mathrm{~F}$ dilator was added to tract dilatation followed by the insertion of a $10 \mathrm{~F}$ pigtail catheter. The catheter was fixed to the abdominal wall skin, placed on gravity drainage for decompression of the infected hydropic gallbladder, and was rinsed daily with $5 \mathrm{ml}$ of sterile saline to ensure the patency of its lumen.

Post-procedural evaluations were performed by cholecystography and tractography after one week of the intervention and before catheter removal. Following a tractography at the end of the fourth week a 0.035 inch guidewire was placed through the catheter needle and the catheter was removed. Over the wire an $8 \mathrm{~F}$ introducer sheath was placed to tract through the skin. A diluted contast agent of $10 \mathrm{ml}$ was injected to check tract maturation. The tract was considered matured in the absence of any contrast leakage to the abdominal cavity. Then the introducer sheath and the guidewire were withdrawn. If there was a contrast leakage, a new catheter of the same diameter was placed into the tract. Tractographic evalution was performed weekly until the maturity of the tract was established (Figure 1).

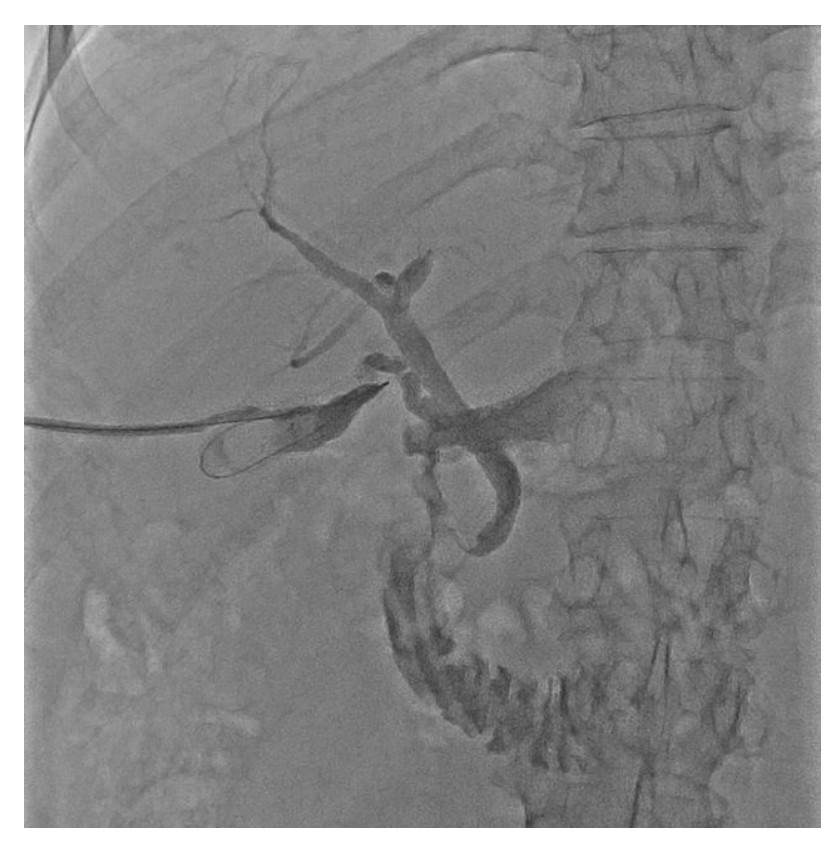

Figure 1: Control tractographic evaluation (After the injection of dilute contrast medium, opacification of the cystic duct, intrahepatic bile ducts, hepatic duct, common bile duct and 
the duedonum without any evidence of contrast leakage through the full length of the cholecystostomy tract demonstrates tract maturity).

\section{Results}

During the three-years time period of our study, 508 patients were admitted to our emergency surgery department with the diagnosis of acute cholecystitis based on the Tokyo guidelines, and initially treated according to the protocol. Conservative treatment was successful in $320(63 \%)$ patients as they were discharged, and referred for elective surgery. Among the remaining 188 $(37 \%)$ cases, $158 \quad(31.1 \%)$ patients underwent emergency surgery while PC was performed in $30 \quad(5.9 \%)$ patients.

Of the 30 patients having undergone PC, $22(73.3 \%)$ were women, whereas 8 $(26.7 \%)$ were men. The mean age was 75.3 (range: 49-99) years. A technically successful PC of the gall bladder via the transhepatic route was performed in all $(100 \%)$ patients under ultrasound guidance. In the PC group; 28 (93.3\%) patients were diagnosed with calculous cholecystitits, while $2(6.7 \%)$ patients possessed acalculous disease.

Five $(16.7 \%)$ patients died from underlying diseases, unrelated to the biliary system, during the follow-up period. Twenty-five $(83.3 \%)$ patients were followed-up. In the PC group, 7 $(23.3 \%)$ patients underwent a subsequent cholecystectomy after 6 weeks, whereas an emergent cholecystectomy was indicated and performed in one $(3.3 \%)$ patient. Two patients $(6.7 \%)$ developed recurrent acute cholecystitis, and were treated with repeated PC without surgery.

\section{Discussion}

Laparoscopic cholecystectomy is considered as the gold standard treatment for patients with symptomatic gallbladder disease. Nevertheless, in an aging subpopulation with medical comorbidities, immediate definitive surgery may not be possible. PC remains to be an alternative method of management for patients with highrisk for being treated with an emergency surgical intervention 7,8 .

The ultrasonography guided PC has been confirmed as a safe and successful management method in a number of publications 9. This minimally invasive procedure has become the preferred initial treatment of choice in high-risk patients who are deemed unsuitable for urgent cholecystectomy. The rationale for PC is based on the principle that initial drainage of the gallbladder palliates acute cholecystitis by permitting acute decompression of the biliary system and subsequent resolution of gallbladder inflammation ${ }^{10}$.

Technically successful placement of the PC was achieved in all $(100 \%)$ of our patients in the PC group. We did not observe any bleeding during insertion although this complication has been documented previously in association with insertion through the transhepatic route ${ }^{11}$. It has also been suggested that there is an increased risk of pneumothorax and empyema when the transhepatic route is used, yet this 
complication was not encountered in our patients, either ${ }^{12,13}$.

For both acute acalculous and calculous cholecystitis, PC is an effective treatment modality with low rates of recurrent disease 14 . When further repeated cholecystitis attacks occur in high risk patients, PC can be repeated as well. In the PC group of our study, two $(6.7 \%)$ patients developed recurrent disease and underwent repeated PCs which were performed successfully without any complications. There are studies that report recurrence rates ranging from $4 \%$ to $22 \%$ after a PC procedure $7,9,15,16$. In contrast, the recurrence rate of the PC group was calculated to be $6.7 \%$ in our study, which was relatively low when compared to the aforementioned studies.

There are studies suggesting that PC may be a definitive therapy for acute acalculous cholecystitis with no need for subsequent elective cholecystectomy ${ }^{17}$. It appears safe to avoid interval cholecystectomy in patients who recover from acalculous cholecystitis, as they are typically highrisk surgical candidates 18 . In the present study, two $(6.7 \%)$ patients were diagnosed with acalculous cholecystitis and PC was performed succesfully.

The disadvantage of a PC is that, although the acute inflammatory events will probably settle, the causative gallbladder stones remain and the patient is at risk of future repeated attacks of cholecystitis. For this reason, elective cholecystectomy is recommended whenever possible 19 .
Subsequent to the management via PC in this study, $8(26.7 \%)$ patients in the PC group underwent a laparoscopic cholecystectomy.

\section{Conclusions}

PC is considered to be a emergency treatment option when conservative treatment of acute cholecystitis fails in the elderly and critically ill patients. PC is a safe approach in surgically high-risk patients with acute cholecystitis and can provide satisfactory long-term results when cholecystectomy is not possible. When further repeated cholecystitis attacks occur, PC can also be repeated in highrisk patients. Tailored surgery should be performed when the patients comorbidities can be optimized.

\section{References}

1. Reynolds W Jr. The first laparoscopic cholecystectomy. JSLS 2001;5:89-94.

2. Hasson HM. Open laparoscopy vs. closed laparoscopy: a comparison of complication rates. Adv Plan Parent 1978;13:41-50.

3. Takada T, Strasberg SM, Solomkin JS, Pitt HA, Gomi H, Yoshida M, et al; Tokyo Guidelines Revision Committee. TG13: Updated Tokyo Guidelines for the management of acute cholangitis and cholecystitis. J Hepatobiliary Pancreat Sci 2013;20:17.

4. Csikesz NG, Tseng JF, Shah SA. Trends in surgical management 
for acute cholecystitis. Surgery 2008;144:283-9.

5. Radder RW. Ultrasonically guided percutaneous catheter drainage for gallbladder empyema. Diagn Imaging 1980;49:330-3.

6. Welschbillig-Meunier K, Pessaux P, Lebigot J, Lermite E, Aube Ch, Brehant $\mathrm{O}$, et al. Percutaneous cholecystostomy for high-risk patients with acute cholecystitis. Surg Endosc 2005;19:1256-9.

7. Li M, Li N, Ji W, Quan Z, Wan X, $\mathrm{Wu} X$, et al. Percutaneous cholecystostomy is a definitive treatment for acute cholecystitis in elderly high-risk patients. Am Surg 2013;79:524-7.

8. Ha JP, Tsui KK, Tang CN, Siu WT, Fung KH, Li MK. Cholecystectomy or not after percutaneous cholecystostomy for acute calculous cholecystitis in high-risk patients. Hepatogastroenterology 2008;55:1497-502.

9. Chang YR, Ahn YJ, Jang JY, Kang MJ, Kwon W, Jung WH, et al. Percutaneous cholecystostomy for acute cholecystitis in patients with high comorbidity and reevaluation of treatment efficacy. Surgery 2014;155:615-22.

10. Macrì A, Scuderi G, Saladino E, Trimarchi G, Terranova M, Versaci $\mathrm{A}$, et al. Acute gallstone cholecystitis in the elderly: treatment with emergency ultrasonographic percutaneous cholecystostomy and interval laparoscopic cholecystectomy. Surg Endosc 2006;20:88-91.

11. Chok KS, Chu FS, Cheung TT, Lam VW, Yuen WK, $\mathrm{Ng} \mathrm{KK}$, et al. Results of percutaneous transhepatic cholecystostomy for high surgical risk patients with acute cholecystitis. ANZ J Surg 2010;80:280-3.

12. Yang KW, Luk WH, Cho DHY, Loke TKL, Chan JCS. A comparison of direct gallbladder puncture with the transhepatic puncture technique in ultrasound-guided cholecystostomy for high surgical risk patients with acute calculous cholecystitis. J HK Coll Radiol 2005;8:222-5.

13. Leveau P, Andersson E, Carlgren I, Willner J, Andersson R. Percutaneous cholecystostomy: a bridge to surgery or definite management of acute cholecystitis in high-risk patients? Scand J Gastroenterol 2008;43:593-6.

14. Kim D, Iqbal SI, Ahari HK, Molgaard CP, Flacke S, Davison BD. Expanding role of percutaneous cholecystostomy and interventional radiology for the management of acute cholecystitis: An analysis of 144 patients. Diagn Interv Imaging. 2017 May 12 Epub. http://dx.doi.org/10.1016/j.diii.2017.04 .006 .

15. McGillicuddy EA, Schuster KM, Barre K, Suarez L, Hall MR, Kaml GJ, et al. Non-operative management of acute cholecystitis in the elderly. $\mathrm{Br} J$ Surg 2012;99:1254-61.

16. Sanjay P, Mittapalli D, Marioud A, White RD, Ram R, Alijani A. Clinical outcomes of a percutaneous cholecystostomy for acute cholecystitis: a multicentre analysis. HPB (Oxford) 2013;15:5116.

17. Soria Aledo V, Galindo Iñíguez L, Flores Funes D, Carrasco Prats M, 
Aguayo Albasini JL. Is cholecystectomy the treatment of choice for acute acalculous cholecystitis? A systematic review of the literature. Rev Esp Enferm Dig 2017;109:708-18.

18. Abbas SH, Ghazanfar MA, GordonWeeks AN, Reddy SR, Soonawalla $Z$, Silva MA. Acalculous Cholecystitis: Is an Elective Interval Cholecystectomy Necessary? Dig
Surg. 2017 Jul 14 Epub. http://dx.doi.org/10.1159/000477780.

19. Silberfein EJ, Zhou W, Kougias P, El Sayed HF, Huynh TT, Albo D, et al. Percutaneous cholecystostomy for acute cholecystitis in high-risk patients: experience of a surgeoninitiated interventional program. Am J Surg 2007;194:672-7. 